\title{
The one-loop divergences of the linear gravity with the torsion terms in tetrad approach.
}

\author{
M. Yu. Kalmykov \\ Bogoliubov Laboratory of Theoretical Physics, Joint Institute for Nuclear Research, \\ 141980 Dubna (Moscow Region), Russian Federation \\ P. I. Proning \\ Department of Theoretical Physics, Physics Faculty \\ Moscow State University, 117234, Moscow, Russian Federation
}

\begin{abstract}
In this paper we discuss the connection between the geometric and tetrad approaches in the quantum affine-metric gravity. The corresponding transition formulas are obtained at the one-loop level. As an example, the one-loop counterterms are calculated in the tetrad formalism in the theory with terms quadratic in the torsion field. This model possesses the extra local symmetries connected with transformation of the connection field. It is shown that the special gauge can be chosen so that the corresponding additional ghosts do not contribute to the one-loop divergent terms.
\end{abstract}

PACS number(s) 0450+h, 0460-m

\footnotetext{
${ }^{1}$ E-mail: kalmykov@thsun1.jinr.dubna.su

${ }^{2}$ E-mail: petr@theor.phys.msu.su
} 


\section{Introduction}

At the present moment there exists no perturbative renormalizable and unitary quantum gravity. All suggested metric models of gravity are nonrenormalizable [1], [2] or nonunitary [3] ones. The known models of the $N=1$ supergravity are finite up to two loops but may generate nonvanishing three-loop divergent counterterms. Models with the extended (e.g. $N=8)$ supersymmetry or some other additional symmetry (e.g. the local conformal symmetry) have better renormalization features, but there is no proof of their complete finiteness by now.

The new type of quantum gravity is connected with a possibility for the quantum (and possible classical) treatment of space-time to involve more than the Riemannian space-time. The most interesting non-Riemannian manifolds are the space-time with torsion [4] and affine-metric space-time [5], [6]. In these geometries, there are geometric objects, additional to the metric tensor, such as torsion and nonmetricity tensors defined as independent variables. At the present moment there are a lot of papers concerning the classical problems of the affine-metric gravity, however, the renormalizability properties of the theory have been studied insufficiently [7]. In particular, these theories possess an additional symmetries connected with the local transformation of the connection fields [8]. Additional global or local symmetries that are maintained at the quantum level without generating anomalies may essentially improve renormalization properties. The influence of these symmetries on the renormalizable properties of the affine-metric quantum gravity is discussed in papers [9]. New hopes for a more perfect quantum gravity arose in connection with string. The discussion of the bosonic string on the affine-metric manifold is given in paper [10].

The affine-metric manifold permits the geometric and tetrad description. The geometric approach implies the description in terms of the metric $g_{\mu \nu}$ and affine connection $\bar{\Gamma}_{\mu \nu}^{\sigma}$. The basic objects are expressed as:

- curvature

$$
\bar{R}_{\lambda \mu \nu}^{\sigma}(\bar{\Gamma})=\partial_{\mu} \bar{\Gamma}_{\lambda \nu}^{\sigma}-\partial_{\nu} \bar{\Gamma}_{\lambda \mu}^{\sigma}+\bar{\Gamma}_{\alpha \mu}^{\sigma} \bar{\Gamma}_{\lambda \nu}^{\alpha}-\bar{\Gamma}_{\alpha \nu}^{\sigma} \bar{\Gamma}_{\lambda \mu}^{\alpha}
$$

- torsion

$$
\bar{Q}_{\mu \nu}^{\sigma}(\bar{\Gamma})=\frac{1}{2}\left(\bar{\Gamma}_{\mu \nu}^{\sigma}-\bar{\Gamma}_{\nu \mu}^{\sigma}\right)
$$

- nonmetricity

$$
\bar{W}_{\sigma \mu \nu}(g, \bar{\Gamma})=\bar{\nabla}_{\sigma} g_{\mu \nu}=\partial_{\sigma} g_{\mu \nu}-\bar{\Gamma}_{\mu \sigma}^{\alpha} g_{\alpha \nu}-\bar{\Gamma}_{\nu \sigma}^{\alpha} g_{\alpha \mu}
$$

In the tetrad formalism, for describing the manifold we use the tetrad $e^{a}{ }_{\mu}$ and the local Lorentz connection $\bar{\Omega}_{b \mu}^{a}$. Using the following relations [6]

$$
\begin{aligned}
g_{\mu \nu} & =e_{\mu}^{a} e_{\nu}^{b} \eta_{a b} \\
\bar{\nabla}_{\sigma} e^{a}{ }_{\mu} & =\partial_{\sigma} e_{\mu}^{a}+\bar{\Omega}_{b \sigma}^{a} e_{\mu}^{b}-\bar{\Gamma}_{\mu \sigma}^{\nu} e_{\nu}^{a}=0
\end{aligned}
$$

where $\eta_{a b}$ is the Minkowskian metric, we can obtain main geometric objects in the tetrad formalism 
- curvature

$$
\bar{R}_{\lambda \mu \nu}^{\sigma}(\bar{\Gamma})=\bar{R}_{b \mu \nu}^{a}(\bar{\Omega}) e_{a}^{\sigma} e_{\lambda}^{b}=\left(\partial_{\mu} \bar{\Omega}_{b \nu}^{a}-\partial_{\nu} \bar{\Omega}_{b \mu}^{a}+\bar{\Omega}_{\alpha \mu}^{a} \bar{\Omega}_{b \nu}^{\alpha}-\bar{\Omega}^{a}{ }_{\alpha \nu} \bar{\Omega}_{b \mu}^{\alpha}\right) e_{a}^{\sigma} e_{\lambda}^{b}
$$

- torsion

$$
\bar{Q}_{\mu \nu}^{\sigma}(\bar{\Gamma})=\bar{Q}_{\mu \nu}^{a}(e, \bar{\Omega}) e_{a}^{\sigma}=-\frac{1}{2}\left(\partial_{\mu} e_{\nu}^{a}-\partial_{\nu} e_{\mu}^{a}+\bar{\Omega}_{b \mu}^{a} e_{\nu}^{b}-\bar{\Omega}_{b \nu}^{a} e_{\mu}^{b}\right) e_{a}^{\sigma}
$$

- nonmetricity

$$
\bar{W}_{\sigma \mu \nu}(g, \bar{\Gamma})=\bar{W}_{\sigma a b}(\bar{\Omega}) e_{\mu}^{a} e_{\nu}^{b}=-\left(\bar{\Omega}_{a b \sigma}+\bar{\Omega}_{b a \sigma}\right) e_{\mu}^{a} e_{\nu}^{b}
$$

The Lagrangian of a gauge theory is built out of terms quadratic in the strength tensor of fields. The curvature is the field-strength tensor of affine and local Lorentz connections. The field-strength tensor for the metric and the tetrad is different. For the former it is the nonmetricity tensor; for the later the torsion tensor. As consequence, the Lagrangian of the affine-metric gravity will have the different form in these two approaches. Nevertheless, this Lagrangian contains about two hundred of arbitrary parameters. It is very difficult to work with such a cumbersome expression. Moreover, some of these coefficients may be equal to zero. We don't know what coefficients are nonzero and only the construction of renormalizable a theory of gravity may answer this question. Hence, any Lagrangians with dimension 2 and 4 terms constructed from the contraction of the curvature, torsion and nonmetricity tensors may be considered as a model of quantum affine-metric gravity.

The number and type of propagating fields depend on the choice of the initial Lagrangian and description approach. In the Lagrangian of the affine-metric quantum gravity fields may exist which are nonpropagating, nondynamical in one formalism (these fields are the nonlinear second class constraints) and propagating, dynamical in another. Due to the unresolved problems of the loop calculations in the theory with second class constraints it is desirable to use the formalism in which all fields are propagating ones. Hence, one needs to have the corresponding quantum transition expressions in order to pass from one formalism to another.

In this paper we will obtain the transition expression (7) from one formalism to another at the quantum level in the affine-metric gravity. As its application we calculate the one-loop counterterms in the theory with terms quadratic in the torsion field in the tetrad formalism. This model possesses the extra local symmetries connected with transformation of the connection field. We will research the influence of this symmetries on the one-loop counterterms.

The following notation and conventions are accepted:

$$
\begin{gathered}
c=\hbar=1 ; \quad \mu, \nu=0,1,2,3 ; \quad a, b=0,1,2,3 ; \quad k^{2}=16 \pi G ; \quad \varepsilon=\frac{4-d}{2} ; \\
e=\operatorname{det}(e) ; \quad(g)=\operatorname{det}\left(g_{\mu \nu}\right),
\end{gathered}
$$


Objects marked by bar are constructed by means of the affine connection $\bar{\Gamma}_{\mu \nu}^{\sigma}$. The others are the Riemannian objects. Parentheses around index pairs denote symmetrization. The Riemannian connection is $\Gamma_{\mu \nu}^{\sigma}=g^{\sigma \lambda} \frac{1}{2}\left(-\partial_{\lambda} g_{\mu \nu}+\partial_{\mu} g_{\lambda \nu}+\partial_{\nu} g_{\lambda \mu}\right)$. For further calculations one needs to define the following tensor object: $D_{\mu \nu}^{\sigma}=\bar{\Gamma}_{\mu \nu}^{\sigma}-\Gamma_{\mu \nu}^{\sigma}$

\section{Connection between the geometric and tetrad ap- proaches at the one-loop level in the affine-metric quantum gravity}

To obtain the corresponding transition expressions at the one loop level let us use the following method: introduce relations (11) and (2) in the initial Lagrangian with the corresponding Lagrange multipliers [6]:

$$
S_{t o t}=S_{g r}(g, \bar{\Gamma}, e, \bar{\Omega})+\frac{N^{(\mu \nu)}}{k^{2}}\left(g_{\mu \nu}-e_{\mu}^{a} e_{\nu}^{b} \eta_{a b}\right)+\frac{M_{a}^{\sigma}{ }_{a}}{k^{2}} \bar{\nabla}_{\sigma} e^{a}{ }_{\mu}
$$

where $S_{g r}(g, \bar{\Gamma}, e, \bar{\Omega})$ may depend on all variables. In this approach relations (11) and (2) are the second class constraints. The Lagrange multiplies are dynamical variables. As a consequence, the Lagrange multiplies may be modified due to loop corrections.

For obtaining the one loop transition expressions we will use the background field method [11]. In accordance with the background field method, all dynamical variables are rewritten as a sum of classical and quantum parts:

$$
\begin{aligned}
& \underline{e}_{\mu}^{a}=e_{\mu}^{a}+k H_{\mu}^{a}, \quad \underline{\Omega}_{b \mu}^{a}=\bar{\Omega}_{b \mu}^{a}+k \omega_{b \mu}^{a}, \\
& \underline{\Gamma}_{\mu \nu}^{\sigma}=\bar{\Gamma}_{\mu \nu}^{\sigma}+k \gamma_{\mu \nu}^{\sigma}, \quad \underline{g}_{\mu \nu}=g_{\mu \nu}+k h_{\mu \nu} \\
& \underline{N}^{\mu \nu}=N^{\mu \nu}+k n^{\mu \nu}, \quad \underline{M}^{\mu}{ }_{a}^{\nu}=M_{a}^{\mu \nu}+k m^{\mu}{ }_{a}{ }^{\nu} \text {. }
\end{aligned}
$$

where $g_{\mu \nu}, \bar{\Gamma}_{\mu \nu}^{\sigma}, e_{\lambda}^{a}, \bar{\Omega}_{b \beta}^{a}, N^{\mu \nu}$ and $M_{a}^{\sigma}{ }_{a}^{\mu}$ are classical parts satisfying the following equation of motion:

$$
\begin{array}{rlrl}
\frac{\delta S_{t o t}}{\delta \bar{\Omega}^{a}{ }_{b \sigma}}=\frac{\delta S}{\delta \bar{\Omega}^{a}{ }_{b \sigma}}+M_{a}^{\sigma b}=0, & & \frac{\delta S_{t o t}}{\delta \bar{\Gamma}^{\nu}{ }_{\mu \sigma}}=\frac{\delta S}{\delta \bar{\Gamma}^{\nu}{ }_{\mu \sigma}}-M_{\nu}^{\sigma}{ }_{\nu}^{\mu}=0 \\
\frac{\delta S_{t o t}}{\delta e^{c}{ }_{\sigma}}=\frac{\delta S}{\delta e^{c}{ }_{\sigma}}-2 N^{(\lambda \sigma)} e_{c \lambda}-\bar{\nabla}_{\lambda} M_{c}^{\lambda \sigma}=0, & \frac{\delta S_{t o t}}{\delta g_{\mu \nu}}=\frac{\delta S}{\delta g_{\mu \nu}}+2 N^{(\mu \nu)}=0 .
\end{array}
$$

where $N^{\mu \nu}$ and $M_{a}^{\sigma}{ }_{a}^{\lambda}$ are the tensor densities.

Expanding the action $S_{\text {tot }}$ in powers of quantum fields up to the terms quadratic in the quantum fields, we obtain the action for the calculation of the one-loop counterterms

$$
\begin{aligned}
S_{t o t}^{(2)} & =n^{\mu \nu}\left(h_{\mu \nu}-H_{\mu \nu}-H_{\nu \mu}\right)-N^{\mu \nu} H_{\mu}^{a} H_{a \nu}+M_{a}^{\sigma \mu}\left(\omega_{b \sigma}^{a} H_{\mu}^{b}-\gamma_{\mu \sigma}^{\nu} H_{\nu}^{a}\right) \\
& +m_{a}^{\sigma}{ }_{a}^{\mu}\left(\bar{\nabla}_{\sigma} H_{\mu}^{a}+\omega_{\mu \sigma}^{a}-\gamma_{\mu \sigma}^{a}\right)+S_{e f f}
\end{aligned}
$$


where $S_{\text {eff }}$ is the action $S(g, \bar{\Gamma}, e, \bar{\Omega})$ quadratic in the quantum fields.

The action $S_{\text {tot }}^{(2)}$ is invariant under the general coordinate transformations

$$
\begin{aligned}
x^{\mu} & \rightarrow{ }^{\prime} x^{\mu}=x^{\mu}+k \xi^{\mu}(x) \\
H^{a}{ }_{\mu}(x) & \rightarrow{ }^{\prime} H^{a}{ }_{\mu}(x)=-\partial_{\mu} \xi^{\nu} e^{a}{ }_{\nu}(x)-\xi^{\nu} \partial_{\nu} e^{a}{ }_{\mu}(x)+O(k) \\
w^{a}{ }_{b \mu}(x) & \rightarrow{ }^{\prime} w^{a}{ }_{b \mu}(x)=-\partial_{\mu} \xi^{\nu} \bar{\Omega}^{a}{ }_{b \nu}(x)-\xi^{\nu} \partial_{\nu} \bar{\Omega}^{a}{ }_{b \mu}(x)+O(k) \\
h_{\mu \nu} & \rightarrow{ }^{\prime} h_{\mu \nu}=h_{\mu \nu}-\nabla_{\mu} \xi_{\nu}-\nabla_{\nu} \xi_{\mu}+O(k) \\
\gamma_{\mu \nu}^{\sigma} & \rightarrow{ }^{\prime} \gamma_{\mu \nu}^{\sigma}=\partial_{\alpha} \xi^{\sigma} \bar{\Gamma}_{\mu \nu}^{\alpha}{ }_{\mu \nu} \partial_{\mu} \xi^{\alpha} \bar{\Gamma}_{\alpha \nu}^{\sigma}-\partial_{\nu} \xi^{\alpha} \bar{\Gamma}_{\mu \alpha}^{\sigma}-\partial_{\mu \nu} \xi^{\sigma}+O(k)
\end{aligned}
$$

and under the local Lorentz rotations

$$
\begin{aligned}
x^{\mu} & \rightarrow{ }^{\prime} x^{\mu}=x^{\mu}+k \Theta^{\mu}{ }_{\nu}(x) x^{\nu} \\
w_{b \mu}^{a}(x) & \rightarrow{ }^{\prime} w_{b \mu}^{a}(x)=\Theta^{a}{ }_{c} \bar{\Omega}^{c}{ }_{b \nu}(x)-\Theta^{c}{ }_{b} \bar{\Omega}^{a}{ }_{c \mu}(x)-\partial_{\mu} \Theta^{a}{ }_{b}+O(k) \\
H^{a}{ }_{\mu}(x) & \rightarrow{ }^{\prime} H^{a}{ }_{\mu}(x)=\Theta^{a}{ }_{b} e^{b}{ }_{\mu}(x)+O(k)
\end{aligned}
$$

The local Lorentz symmetry is fixed by means of the following gauge [2]:

$$
H_{[\mu \nu]}=0
$$

To violate the general coordinate transformation, we use the following gauge condition:

$$
\begin{aligned}
f^{\mu} & =T^{\mu \sigma(\alpha \beta)} \nabla_{\sigma} h_{\alpha \beta}+\left(E_{\sigma}^{\mu \rho \lambda \alpha \beta} \nabla_{\rho} \nabla_{\lambda}+G_{\sigma}^{\mu \alpha \beta}\right) \gamma_{\alpha \beta}^{\sigma}+K^{\mu \sigma(\alpha \beta)} \nabla_{\sigma} H_{(\alpha \beta)} \\
L_{g f} & =\frac{1}{2 \zeta} f^{\mu} \chi_{\mu \nu} f^{\nu}
\end{aligned}
$$

where $\chi_{\mu \nu}$ is the differential operator; $T^{\mu \sigma(\alpha \beta)}, K^{\mu \sigma(\alpha \beta)} E^{\mu \rho \lambda \alpha}{ }_{\sigma}^{\alpha \beta}$ and $G_{\sigma}^{\mu \alpha}$ are the tensors depending on the background fields, $\zeta$ is the gauge parameter. The ghost action is

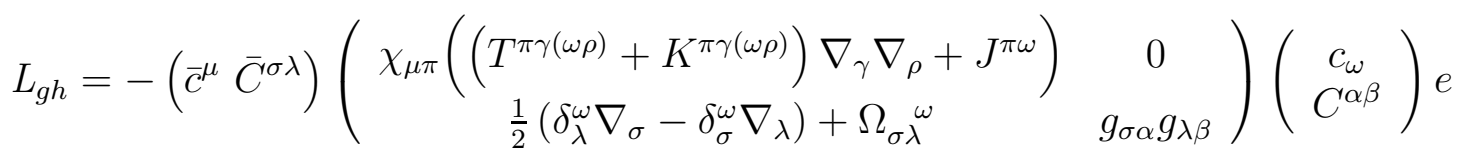

where $\left\{\bar{c}^{\mu}, c^{\nu}\right\}$ and $\left\{\bar{C}^{\sigma \lambda}, C^{\mu \nu}\right\}$ are the ghost fields connected with the general coordinate and local Lorentz transformations and

$$
\begin{aligned}
J_{\omega}^{\mu} & =\left(E_{\sigma}^{\mu \rho \lambda \alpha \beta} \nabla_{\rho} \nabla_{\lambda}+G_{\sigma}^{\mu \alpha \beta}\right)\left(\delta_{\omega}^{\sigma} D_{\alpha \beta}^{\tau} \nabla_{\tau}-D_{\omega \beta}^{\sigma} \nabla_{\alpha}-D_{\alpha \omega}^{\sigma} \nabla_{\beta}-\nabla_{\omega} D_{\alpha \beta}^{\sigma}\right. \\
& \left.-\frac{1}{2}\left(\nabla_{\alpha} \nabla_{\beta}+\nabla_{\beta} \nabla_{\alpha}\right) \delta_{\omega}^{\sigma}+\frac{1}{2}\left(R_{\alpha \beta \omega}^{\sigma}+R_{\beta \alpha \omega}^{\sigma}\right)\right)
\end{aligned}
$$

The gauge fixing term with higher derivatives may break the unitary of the theory. To avoid this problem we consider the case $E_{\sigma}^{\mu \rho \lambda \alpha \beta}=0$. Introducing the notations $s_{\mu \nu}=\frac{1}{2}\left(H_{\mu \nu}+H_{\nu \mu}\right)$ and $t_{\mu \nu}=\frac{1}{2}\left(H_{\mu \nu}-H_{\nu \mu}\right)$ we have 


$$
\begin{aligned}
e^{i W}= & \int \exp \left(S_{e f f}(g, \bar{\Gamma}, e, \bar{\Omega})+S_{g h}+S_{g f}+M_{a}^{\sigma \mu}\left(\omega_{b}^{a}{ }_{b} H_{\mu}^{b}-\gamma_{\mu \sigma}^{\nu} H_{\nu}^{a}\right)\right. \\
& \left.-N^{\mu \nu} H_{\mu}^{a} H_{a \nu}\right) d h_{\alpha \beta} d \gamma_{\mu \nu}^{\sigma} d s_{\mu \nu} d t_{\alpha \beta} d \omega^{a}{ }_{b \mu} d \bar{c}^{\mu} d c^{\nu} d \bar{C}^{\sigma \lambda} d C^{\mu \nu} \\
& \delta\left(h_{\mu \nu}-2 s_{\mu \nu}\right) \delta\left(t_{\alpha \beta}\right) \delta\left(\bar{\nabla}_{\sigma} s_{\mu}^{a}+\omega_{\mu \sigma}^{a}-\gamma_{\mu \sigma}^{a}\right)\left(\operatorname{det} \chi_{\mu \nu}\right)^{\frac{1}{2}}
\end{aligned}
$$

Let us write the action $S_{g r}(g, \bar{\Gamma}, e, \bar{\Omega})$ of the affine-metric gravity either in the geometric formalism $(g, \bar{\Gamma})$ or tetrad approach $(e, \bar{\Omega})$. Then, from the equation of motion (5) we obtain

$$
M_{a}^{\sigma \lambda}=N^{\mu \nu}=0
$$

The contribution of the Lorentz ghosts in the effective action is trivial in the gauge (6) in the dimensional regularization. The one-loop generating functional is :

$$
\begin{aligned}
e^{i W}= & \int \exp \left(S_{e f f}(g, \bar{\Gamma}, e, \bar{\Omega})+\frac{1}{2 \zeta} f^{\mu} \chi_{\mu \nu} f^{\nu}\right) d h_{\alpha \beta} d \gamma_{\mu \nu}^{\sigma} d s_{\mu \nu} d t_{\alpha \beta} d \omega_{b \mu}^{a} d \bar{c}^{\mu} d c^{\nu} \\
& \operatorname{det}\left(\chi_{\mu \pi}\left(\left(T^{\pi \gamma(\omega \rho)}+K^{\pi \gamma(\omega \rho)}\right) \nabla_{\gamma} \nabla_{\omega}+J_{\rho}^{\pi}\right)\right) \\
& \delta\left(h_{\mu \nu}-2 s_{\mu \nu}\right) \delta\left(t_{\alpha \beta}\right) \delta\left(\bar{\nabla}_{\sigma} s_{\mu}^{a}+\omega_{\mu \sigma}^{a}-\gamma_{\mu \sigma}^{a}\right)\left(\operatorname{det} \chi_{\mu \nu}\right)^{\frac{1}{2}}
\end{aligned}
$$

Hence, in the gauge (6) the transition expressions from one formalism to another are

$$
\begin{aligned}
h_{\mu \nu} & =2 H_{(\mu \nu)} \\
\gamma_{(\mu \nu) \sigma} & =\omega_{(\mu \nu) \sigma}+\bar{\nabla}_{\sigma} H_{(\mu \nu)} \\
\gamma_{[\mu \nu] \sigma} & =\omega_{[\mu \nu] \sigma} \\
H_{[\mu \nu]} & =0
\end{aligned}
$$

The contribution of the ghost fields to the one loop counterterms in the gauge (6) is independent of the choice of a formalism.

\section{The linear gravity with the torsion terms}

Consider a simple model with the terms quadratic in the torsion fields. The Lagrangian of the model is the following:

$$
S_{g r}=-\frac{1}{k^{2}} \int d^{4} x \sqrt{-g}\left(\bar{R}(\bar{\Gamma})-2 \Lambda+b_{1} \bar{Q}_{\sigma \mu \nu} \bar{Q}^{\sigma \mu \nu}+b_{2} \bar{Q}_{\sigma \mu \nu} \bar{Q}^{\nu \mu \sigma}+b_{3} \bar{Q}_{\sigma} \bar{Q}^{\sigma}\right)
$$

where $\Lambda$ is a cosmological constant, $\left\{b_{i}\right\}$ are arbitrary constants and $\bar{Q}_{\sigma}=\bar{Q}_{\sigma \lambda}^{\lambda}$

The torsion tensor has a different meaning within the geometric and tetrad formalisms. In the tetrad approach the torsion tensor is the strength tensor of tetrad 
fields, whereas in the geometric approach the torsion tensor plays an auxiliary role. Let us calculate the one-loop counterterms for the model (8) within the tetrad approach where $e^{a}{ }_{\mu}$ and $\bar{\Omega}_{b \sigma}^{a}$ are independent dynamical variables. For calculating the one-loop counterterms we will use the background-field method [11] and expressions (7).

In the case of special choice of the coefficients $\left\{b_{j}\right\}$ the action (8) is invariant under the extra local transformations of the connection field [8]. This extra invariance is the sum of the projective invariance:

$$
\Gamma_{\mu \nu}^{\sigma}(x) \rightarrow{ }^{\prime} \Gamma_{\mu \nu}^{\sigma}(x)=\Gamma_{\mu \nu}^{\sigma}(x)+k \delta_{\mu}^{\sigma} C_{\nu}(x)
$$

and antisymmetric one

$$
\begin{aligned}
x^{\mu} & \rightarrow{ }^{\prime} x^{\mu}=x^{\mu} \\
g_{\mu \nu}(x) & \rightarrow{ }^{\prime} g_{\mu \nu}(x)=g_{\mu \nu}(x) \\
\Gamma_{\mu \nu}^{\sigma}(x) & \rightarrow{ }^{\prime} \Gamma_{\mu \nu}^{\sigma}(x)=\Gamma_{\mu \nu}^{\sigma}(x)+k g^{\sigma \lambda} I_{[\lambda \mu \nu]}(x)
\end{aligned}
$$

where $C_{\nu}(x)$ and $I_{\lambda \mu \nu}(x)$ are an arbitrary vector and antisymmetric tensor respectively. These invariances (9) and (10) arises from the following choice of coefficients $\left\{b_{j}\right\}$ :

$$
b_{2}=b_{1}-1, \quad b_{3}=\frac{1}{3}-b_{1}
$$

The classical equations of motion are:

$$
\begin{aligned}
R_{\mu \nu} & =\Lambda g_{\mu \nu} \\
D_{\mu \nu}^{\sigma} & =0
\end{aligned}
$$

We will consider the theory with the additional invariances (9) and (10). For simplicity we will calculate the one-loop counterterms on shell (11).

The one-loop Lagrangian on-shell quadratic in the quantum fields is

$$
L_{e f f}=-\frac{1}{2} \gamma_{\mu \nu}^{\sigma} F_{\sigma \lambda}^{\mu \nu}{ }_{\lambda}^{\alpha \beta} \gamma_{\alpha \beta}^{\lambda}-\frac{1}{2} h^{\alpha \beta} X_{\alpha \beta \mu \nu} h^{\mu \nu}-\gamma_{\alpha \beta}^{\lambda} B_{\lambda}^{\alpha \beta \sigma} \nabla_{\sigma} h^{\mu \nu}
$$

where

$$
\begin{aligned}
& F_{\alpha}^{\beta \lambda \nu}= g^{\beta \lambda} \delta_{\alpha}^{\nu} \delta_{\mu}^{\sigma}+g^{\nu \sigma} \delta_{\alpha}^{\lambda} \delta_{\mu}^{\beta}-b_{1} g^{\beta \sigma} \delta_{\alpha}^{\nu} \delta_{\mu}^{\lambda}-b_{1} g^{\lambda \nu} \delta_{\alpha}^{\sigma} \delta_{\mu}^{\beta} \\
&+ b_{1} g_{\alpha \mu} g^{\beta \nu} g^{\sigma \lambda}-b_{1} g_{\alpha \mu} g^{\lambda \nu} g^{\beta \sigma}+\frac{b_{1}-1}{2}\left(g^{\lambda \sigma} \delta_{\mu}^{\beta} \delta_{\alpha}^{\nu}+g^{\beta \nu} \delta_{\mu}^{\lambda} \delta_{\alpha}^{\sigma}\right) \\
&+ \frac{1-3 b_{1}}{6}\left(g^{\beta \nu} \delta_{\alpha}^{\lambda} \delta_{\mu}^{\sigma}-g^{\beta \sigma} \delta_{\mu}^{\nu} \delta_{\alpha}^{\lambda}-g^{\lambda \nu} \delta_{\alpha}^{\beta} \delta_{\mu}^{\sigma}+g^{\sigma \lambda} \delta_{\alpha}^{\beta} \delta_{\mu}^{\nu}\right) \\
& P^{\alpha \beta \mu \nu}= \frac{1}{4}\left(g^{\alpha \mu} g^{\beta \nu}+g^{\alpha \nu} g^{\beta \mu}-g^{\alpha \beta} g^{\mu \nu}\right) \\
& B_{\lambda}^{\alpha \beta \sigma}=2\left(\delta_{\lambda}^{\sigma} P_{\mu \nu}^{\alpha \beta}-\delta_{\lambda}^{\beta} P_{\mu \nu}^{\alpha \sigma}\right) \\
& X_{\alpha \beta \mu \nu}=2 \Lambda P_{\alpha \beta \mu \nu}
\end{aligned}
$$


This expression was obtained in the geometric formalism. We can rewrite the Lagrangian (12) in the tetrad formalism using expressions (7). For further calculations let us introduce the following notation: $\omega_{\sigma \mu \nu}=\omega_{[\sigma \mu] \nu}+\omega_{(\sigma \mu) \nu} \equiv p_{[\sigma \mu] \nu}+l_{(\sigma \mu) \nu}$. Then,

$$
\begin{aligned}
L_{e f f}= & \left(-\frac{1}{2} p_{\mu \nu}^{\sigma} F_{\sigma \lambda}^{(3) \mu \nu}{ }_{\alpha \beta} p_{\alpha \beta}^{\lambda}-2 H^{\alpha \beta} H^{\mu \nu} X_{\alpha \beta \mu \nu}-2 p_{\alpha \beta}^{\lambda} B_{\lambda}^{\alpha \beta \sigma}{ }_{\mu \nu} \nabla_{\sigma} H^{\mu \nu}\right. \\
& \left.-\frac{1}{2}\left(l_{\mu \nu}^{\sigma}+\nabla_{\nu} H_{\mu}^{\sigma}\right) F_{\sigma \lambda}^{(4) \mu \nu}{ }_{\alpha \beta}{ }^{\alpha \beta} l_{\alpha \beta}^{\lambda}+\nabla_{\beta} H_{\alpha}^{\lambda}\right) \\
& \left.-\left(l_{\alpha \beta}^{\lambda}+\nabla_{\beta} H_{\alpha}^{\lambda}\right) 2 B_{\lambda}^{\alpha \beta \sigma}{ }_{\mu \nu} \nabla_{\sigma} H^{\mu \nu}\right) \sqrt{-g}
\end{aligned}
$$

where $\omega_{\sigma \mu \nu}$ and $H_{\mu \nu}$ are defined in (四) and

$$
F^{(3) \sigma \mu \nu \lambda \alpha \beta}=F^{[\sigma \mu] \nu[\lambda \alpha] \beta}, \quad F^{(4) \sigma \mu \nu \lambda \alpha \beta}=F^{(\sigma \mu) \nu(\lambda \alpha) \beta}
$$

Expression (13) is expansion of the initial Lagrangian (8) written in the tetrad formalism up to terms quadratic in the quantum fields with the local Lorentz connection $\Omega_{b \sigma}^{a}$ and tetrad $e_{\lambda}^{a}$ as independent dynamical variables. In this expression the local Lorentz symmetry is broken by the condition (6). To define the propagator of the quantum field $\omega_{\mu \nu}^{\sigma}\left(\gamma_{\mu \nu}^{\sigma}\right)$ we must fix the additional symmetries (9) and (10) at the quantum level. The gauge conditions are

$$
\begin{aligned}
f_{\lambda}^{(1)} & =A_{1} \delta_{\lambda}^{\beta} \delta_{\sigma}^{\alpha} \gamma_{\alpha \beta}^{\sigma}=A_{1} \delta_{\lambda}^{\beta} \delta_{\sigma}^{\alpha}\left(\omega_{\alpha \beta}^{\sigma}+\nabla_{\beta} H_{\alpha}^{\sigma}\right) \\
f^{(2)^{\lambda}} & =A_{2} \varepsilon^{\lambda \sigma \alpha \beta} \gamma_{\sigma \alpha \beta}=A_{2} \varepsilon^{\lambda \sigma \alpha \beta} \omega_{\sigma \alpha \beta} \\
L_{g f}^{a d d} & =\frac{1}{2} f^{(1) \lambda} f_{\lambda}^{(1)}+\frac{1}{2} f^{(2) \lambda} f_{\lambda}^{(2)} ;
\end{aligned}
$$

where the constants $A_{1}$ and $A_{2}$ are nonzero. We violate the coordinate invariance of the action by means of the following gauge:

$$
\begin{aligned}
F_{\mu} & =\nabla_{\nu} H_{\mu}^{\nu}-\frac{1}{2} \nabla_{\mu} H_{\alpha}^{\alpha} \\
L_{g f}^{\text {coor }} & =2 F_{\mu} F^{\mu}
\end{aligned}
$$

The corresponding one-loop ghost on-shell Lagrangian is

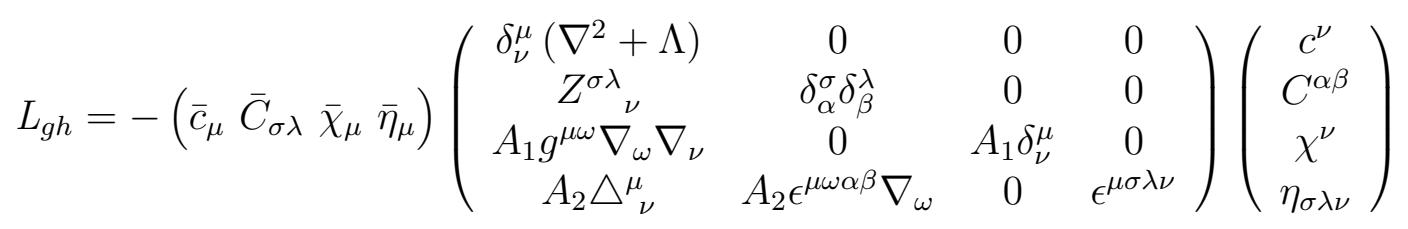

where $\left\{\bar{c}_{\nu}, c^{\mu},\right\},\left\{\bar{C}_{\alpha \beta}, C^{\mu \nu}\right\}, \quad\left\{\bar{\chi}_{\alpha}, \chi^{\beta}\right\},\left\{\bar{\eta}_{\sigma}, \eta_{\mu \nu}^{\lambda}\right\}$ are anticommuting ghost fields connected with the general coordinate, local Lorentz, projective and antisymmetric transformations, respectively. $\triangle^{\mu}{ }_{\nu}$ and $Z_{\nu}^{\mu}$ are 


$$
\begin{gathered}
Z_{\sigma \lambda}{ }^{\nu}=\frac{1}{2}\left(\delta_{\sigma}^{\nu} \nabla_{\lambda}-\delta_{\lambda}^{\nu} \nabla_{\sigma}\right)-\Omega_{\sigma \lambda}{ }^{\nu} \\
\triangle_{\nu}^{\mu}=\epsilon^{\mu \lambda \alpha \beta}\left(\Omega_{\alpha \beta \nu} \nabla_{\lambda}+\nabla_{\nu} \Omega_{\alpha \beta \lambda}+\Omega_{\alpha c \lambda} \Omega_{\beta \nu}^{c}{ }_{\beta \nu}-\Omega_{\alpha c \nu} \Omega_{\beta \lambda}^{c}\right)
\end{gathered}
$$

After inessential redefinition of the ghost fields we obtain that the one-loop contribution of the $\left\{\bar{C}_{\alpha \beta}, C^{\mu \nu}\right\},\left\{\bar{\chi}_{\alpha}, \chi^{\beta}\right\},\left\{\bar{\eta}_{\sigma}, \eta_{\mu \nu}^{\lambda}\right\}$ ghosts to the effective action is proportional to $\delta^{4}(0)$. In the dimensional regularization [12], $\left[\delta^{4}(0)\right]_{R}=0$ and the contribution of these ghosts to the one-loop counterterms is equal to zero.

Replace the dynamical variables in the following way:

$$
\begin{aligned}
& \tilde{p}_{\mu \nu}^{\sigma}=p_{\mu \nu}^{\sigma}+2 \bar{F}_{\mu \nu \alpha \beta}^{-1(3) \sigma}{ }_{\lambda{ }_{\lambda \epsilon}^{\lambda}}^{\alpha \beta \tau} \nabla_{\tau} H^{\rho \epsilon} \\
& \tilde{l}_{\mu \nu}^{\sigma}=l_{\mu \nu}^{\sigma}+\nabla_{\nu} H_{\mu}^{\sigma}+2 \bar{F}_{\mu \nu \alpha \beta}^{-1(4) \sigma}{ }_{\lambda}^{\lambda}{ }_{\rho \epsilon}^{\alpha \beta \tau} \nabla_{\tau} H^{\rho \epsilon}
\end{aligned}
$$

where

$$
\bar{F}^{-1(3) \sigma \mu \nu \lambda \alpha \beta}=\bar{F}^{-1[\sigma \mu] \nu[\lambda \alpha] \beta}, \quad \bar{F}^{-1(4) \sigma \mu \nu \lambda \alpha \beta}=\bar{F}^{-1(\sigma \mu) \nu(\lambda \alpha) \beta}
$$

and

$$
\begin{aligned}
\bar{F}_{\beta \sigma \nu \lambda}^{-1 \alpha \mu}= & -\frac{1}{4} g^{\alpha \mu} g_{\beta \sigma} g_{\nu \lambda}+\left(\frac{1}{4}+\frac{1}{9 b_{1}}+\frac{1}{36 A_{2}}\right) g^{\alpha \mu} g_{\beta \nu} g_{\sigma \lambda}-\left(\frac{1}{12}+\frac{1}{18 b_{1}}\right) g_{\nu \beta} \delta_{\lambda}^{\mu} \delta_{\sigma}^{\alpha} \\
& +\frac{1}{4}\left(g_{\nu \lambda} \delta_{\beta}^{\mu} \delta_{\sigma}^{\alpha}+g_{\beta \sigma} \delta_{\nu}^{\alpha} \delta_{\lambda}^{\mu}\right)-\left(\frac{1}{4}+\frac{1}{18 b_{1}}-\frac{1}{36 A_{2}}\right)\left(g_{\nu \sigma} \delta_{\lambda}^{\alpha} \delta_{\beta}^{\mu}+g_{\beta \lambda} \delta_{\sigma}^{\mu} \delta_{\nu}^{\alpha}\right) \\
& +\frac{1}{8}\left(g_{\nu \lambda} \delta_{\sigma}^{\mu} \delta_{\beta}^{\alpha}+g_{\beta \sigma} \delta_{\lambda}^{\alpha} \delta_{\nu}^{\mu}\right)+\left(\frac{1}{4}-\frac{1}{9 b_{1}}-\frac{1}{36 A_{2}}\right) g^{\alpha \mu} g_{\sigma \nu} g_{\beta \lambda} \\
& -\left(\frac{1}{24}-\frac{1}{18 b_{1}}\right)\left(g_{\beta \lambda} \delta_{\nu}^{\mu} \delta_{\sigma}^{\alpha}+g_{\sigma \nu} \delta_{\beta}^{\alpha} \delta_{\lambda}^{\mu}\right)-\left(\frac{1}{48}+\frac{1}{18 b_{1}}-\frac{1}{16 A_{1}}\right) g_{\sigma \lambda} \delta_{\nu}^{\mu} \delta_{\beta}^{\alpha} \\
& -\left(\frac{1}{4}-\frac{1}{18 b_{1}}+\frac{1}{36 A_{2}}\right)\left(g_{\nu \beta} \delta_{\sigma}^{\mu} \delta_{\lambda}^{\alpha}+g_{\sigma \lambda} \delta_{\beta}^{\mu} \delta_{\nu}^{\alpha}\right)
\end{aligned}
$$

and $b_{1} \neq 0$.

The replacement (17) does not change the functional measure

$$
\operatorname{det}\left|\frac{\partial(H, \tilde{l}, \tilde{p})}{\partial(H, l, p)}\right|=1
$$

In the new variables the action (13) is the following:

$$
\begin{aligned}
L_{e f f} & =\left(-\frac{1}{2} \tilde{p}_{\mu \nu}^{\sigma} \bar{F}_{\sigma \lambda}^{(3) \mu \nu \alpha \beta} \tilde{p}_{\alpha \beta}^{\lambda}-\frac{1}{2} \tilde{l}_{\mu \nu}^{\sigma} \bar{F}_{\sigma \lambda}^{(4) \mu \nu}{ }_{\alpha \beta}^{\alpha \beta} \tilde{l}_{\alpha \beta}^{\lambda}-2 H^{\mu \nu} H^{\sigma \lambda}\left(R_{\mu \sigma \nu \lambda}-R_{\mu \sigma} g_{\nu \lambda}\right)\right. \\
& +\nabla_{\sigma} H_{\alpha \beta} \nabla_{\lambda} H^{\alpha \beta} g^{\sigma \lambda}-2 \nabla_{\mu} H_{\nu}^{\mu} \nabla_{\lambda} H^{\lambda \nu}+2 \nabla_{\mu} H_{\sigma}^{\sigma} \nabla_{\nu} H^{\mu \nu}-\nabla_{\sigma} H_{\mu}^{\mu} \nabla_{\lambda} H_{\nu}^{\nu} g^{\sigma \lambda} \\
& \left.-2 H^{\alpha \beta} H^{\mu \nu} X_{\alpha \beta \mu \nu}\right) e
\end{aligned}
$$


where $\bar{F} \underset{\sigma{ }_{\lambda}}{\alpha \beta \mu \nu}=F_{\sigma \lambda}^{\alpha \beta \mu \nu}+f^{(1) \lambda} f_{\lambda}^{(1)}+f^{(2) \lambda} f_{\lambda}^{(2)}$.

The one-loop counterterms on the mass-shell including the contributions of the quantum and ghost fields are

$$
\triangle \Gamma_{\infty}^{(1)}=-\frac{1}{32 \pi^{2} \varepsilon} \int d^{4} x \sqrt{-g}\left(\frac{53}{45} R_{\alpha \beta \mu \nu} R^{\alpha \beta \mu \nu}-\frac{58}{5} \Lambda^{2}\right)
$$

\section{Conclusions}

In the present paper we have obtained the transition expressions (7) which connect the geometric and tetrad approaches at the one-loop level within the background formalism. Using these expressions one-loop quantum corrections and renormalization properties of the affine-metric quantum gravity can be investigated in an arbitrary formalism. The essential step for finding of these transition expressions is the gauge (6) fixing the local Lorentz invariance.

Let us consider the model (8) with extra local symmetries (9) and (10).

1. The projective (9) and antisymmetric (10) invariances do not influence on the renormalizability of the model (8).

2. In the model (8) the terms which are quadratic over the torsion fields do not contribute to the one-loop counterterms neither in the geometric nor in the tetrad approach. So, we can assert, that in the theory with quadratic in the torsion and nonmetricity Lagrangian these fields play an auxiliary role at the quantum level, violating the extra local symmetries of the affine-metric gravity.

3. The special gauges $((14)$ and (15)) are found so that the corresponding action for ghosts related to the extra local symmetries transformations has an algebraic form. In any invariant regularizaton with $[\delta(0)]_{\text {ren }}=0$ its contribution into oneloop counterterms is zero.

4. The additional condition $\left(b_{1} \neq 0\right)$ arising in the definition of connection field propagator (18) corresponds to the new symmetry of our theory. We do not know how the connection field transforms under this new symmetry. Only point we know exactly is the metric and matter fields are invariant under these transformations.

5. The theory involved is renormalizable at the one-loop level on the mass-shell.

\section{References}

[1] G. 't Hooft and M. Veltman, Ann. Inst. H. Poincaré A 20 (1974) 69;

M. H. Goroff and A. Sagnotti, Nucl. Phys. B 266 (1986) 709.

[2] S. Deser and P. van Nieuwenhuizen, Phys. Rev. D 10 (1974) 411.

[3] K. S. Stelle, Phys. Rev. D 16 (1977) 953. 
[4] F. W. Hehl, P. van der Heyde, G. D. Kerlick and I. M. Nester, Rev. Mod. Phys. 48 (1976) 393;

K. Hayashi and T. Shirafyji, Prog. Theor. Phys. 64 (1980) 866, 883, 1435, 2222; ibid 65 (1981) 525;

F. W. Hehl, Found. Phys. 15 (1985) 451.

[5] L. Smolin, Nucl. Phys. B 247 (1984) 511;

J. Dell, J. L. deLyra and L. Smolin, Phys. Rev. D 34 (1986) 3012;

Y. Ne'eman and D. Šijački, Phys. Lett. B 200 (1988) 489;

F. W. Hehl, J. D. McCrea, E. W. Mielke and Y. Ne'emann, Found. Phys. 19 (1989) 1075.

[6] F. W. Hehl, J. D. McCrea, E. W. Mielke and Y. Ne'emann, Phys. Rep. 258 (1995) 1 and References therein.

[7] C. Y. Lee and Y. Ne'eman, Phys. Lett. B 233 (1989) 286; ibid, B 242 (1990) 59; C. Y. Lee, Class. Quant. Grav. 9 (1992) 2001.

[8] V. D. Sandberg, Phys. Rev. D 12 (1975) 3013.

[9] M. Yu. Kalmykov and P. I. Pronin, Nuovo Cimento B 106 (1991) 1401; Gen. Rel. Grav. 27 (1995) 873;

M. Yu. Kalmykov, P. I. Pronin and K. V. Stepanyantz, Class. Quant. Grav. 11 (1994) 2645;

M. Yu. Kalmykov, Class. Quant. Grav. 14 (1997) 367.

[10] V. E. Tarasov, Phys. Lett. B 323 (1994) 296.

[11] B. S. DeWitt, Dynamical Theory Groups and Fields (Gordon and Breach, New York, 1965).

[12] G. 't Hooft and M. Veltman, Nucl. Phys. B 44 (1972) 189. 